\title{
Human Sex Matters: Y-linked lysine demethylase 5D drives accelerated male
}

Madlen Merten ${ }^{1, *}$, Johannes F.W. Greiner ${ }^{2,3, *}$, Tarek Niemann ${ }^{1,3}$, Meike Grosse Venhaus ${ }^{1}$,

${ }^{1}$ Molecular Neurobiology, Bielefeld University, Universitätsstrasse 25, 33615 Bielefeld, Germany;

${ }^{2}$ Department of Cell Biology, Bielefeld University, Universitätsstrasse 25, 33615 Bielefeld, Germany;

${ }^{3}$ Forschungsverbund BioMedizin Bielefeld FBMB e.V., Bielefeld, Germany

${ }^{4}$ Department of Regenerative Musculoskeletal Medicine, Institute for Musculoskeletal Medicine,

Campus 1, Building D3, 48149 Muenster, Germany;

${ }^{5}$ Department of Trauma and Orthopedic Surgery, Protestant Hospital of Bethel Foundation, University

\# Correspondence: barbara.kaltschmidt@uni-bielefeld.de; Tel.: +49-521-106-5624

* Authors contributed equally to this work.

${ }^{\S}$ Authors contributed equally to this work.

Running Head: KDM5D drives sexually dimorphic osteogenic differentiation 
23

\section{Abstract}

Female sex is increasingly associated to a loss of bone mass during aging and an increased risk for fractures developing nonunion. Hormonal factors and cell-intrinsic mechanisms are suggested to drive these sexual dimorphisms, although underlying molecular mechanisms are still a matter of debate. Here, we observed a decreased capacity of calvarial bone recovery in female rats and a profound sexually dimorphic osteogenic differentiation human adult neural crest-derived stem cells (NCSCs). Next to an elevated expression of pro-osteogenic regulators, global trancriptomics revealed Lysine Demethylase 5D (KDM5D) to be highly upregulated in differentiating male NCSCs. Loss of function by siRNA or pharmacological inhibition of KDM5D significantly reduced the osteogenic differentiation capacity of male NCSCs. In summary, we demonstrate craniofacial osteogenic differentiation to be sexually dimorphic with the expression of KDM5D as a prerequisite for accelerated male osteogenic differentiation, emphasizing the analysis of sex-specific differences as a crucial parameter for treating bone defects. 


\section{Introduction}

The capacity for bone regeneration as well as the severity and occurrence of bone disorders is increasingly noticed to be linked to the sex of an individual (1), reviewed in (2). Here, particularly female sex is linked to a gradual loss of bone mass after the age of around $35(3,4)$ and considered as a major risk factor for decreased osseointegration of implants (5) and fractures developing nonunion $(1,6)$. Postmenopausal women also show an increased prevalence of osteoporosis compared to their male counterparts (7-9). While aggressive osteoclastic bone resorption was recently reported to be linked to age and menopausal status of female individuals (4), the bone formation rate simultaneously decreases $(4,9)$. On mechanistic level, estrogen was reported to regulate bone resorption and formation (10) and the osteogenic activity of stem cells in mouse osteoporosis models (11). However, Meszaros and colleagues reported a significantly increased regeneration of cranial bone defects in castrated or non-operated male mice compared to non-operated or ovariectomized female animals, suggesting the additional presence of non-hormonal mechanisms guiding sexual dimorphic bone regeneration rates (12). In this line, increasing evidences suggest that stem cell-intrinsic mechanisms account for sex-specific differences in bone recovery and occurrence of bone disorders (reviewed in (2)). For instance, male mouse skeletal muscle-derived stem cells were reported to reveal a greater amount of mineralization and ALP-activity after osteogenic differentiation compared to their female counterparts (13). In humans, male adipose tissue stem cells (ASCs) were likewise shown to more efficiently give rise to osteogenic derivates compared to female ASCs (14). Despite these promising findings, the molecular mechanisms of the observed sex-specific differences in osteogenic differentiation of stem cells, particularly in the cranial and craniofacial region, are still a matter of debate.

Facing this challenge, the present study demonstrates sex-specific differences in the regeneration of critical size calvarial rat bone defects as well as in the osteogenic differentiation of adult human craniofacial stem cells and determines underlying molecular drivers regulating sex-specific stem cell behavior. We particularly utilized adult human neural crest-derived stem cells (NCSCs) from the nasal cavity as a human in vitro model for investigating sexual dimorphisms in craniofacial osteogenic differentiation in accordance to our previous studies (15-17). For guiding osteogenic differentiation of NCSCs, collagen type I fibers harboring $30 \mathrm{~nm}$ pores were applied as a pro-osteogenic biophysical cue (16). Next to collagen type I fibers, the clinically approved hemostatic collagen sponge Spongostan was utilized for investigating the sexual dimorphic regeneration of critical size calvarial rat bone defects. We recently demonstrated the distinct nano- and microtopography of Spongostan to be solely sufficient for guiding bone recovery in critical size calvarial bone defects in male rats $(18,19)$. Taking advantage of these biophysical cues driving osteogenic differentiation, we show here an elevated 
regeneration of critical size calvarial bone defects in male rats after transplantation of collagen fibers or Spongostan compared to their female counterparts. In accordance to our observations in vivo, human NCSCs from female individuals revealed a strong delay in osteogenic differentiation on collagen type I fibers over time in comparison to NCSCs from male donors. Global gene expression profiling following osteogenic differentiation revealed a sex-specific expression pattern of negative osteogenic regulators like micro RNA 137 (MIR137HG) and taxilin gamma (TXLNG) in female NCSCs, while proosteogenic regulators including C-FOS and Cytochrome P450 Family 7 Subfamily B Member 1 (CYP7B1) were upregulated in male stem cells. We further functionally validated the expression of the Y-linked 
84

\section{Results}

\section{Calvarial critical size defects in female rats show impaired regeneration compared to male animals}

We determined potential differences in bone recovery between the sexes by comparing regeneration of critical-size calvarial bone defects in male and female rats. To allow recovery of the lesion, we applied the collagen type I fibers or the collagen sponge Spongostan in accordance to our previous observations $(18,19)$ after trepanation of calvarial bones. Although no closures of the critical-size defects were observable in controls via micro-computed tomography $(\mu \mathrm{CT})$, we observed a significantly reduced bone mineral density (BMD) in female rats compared to male ones (Fig. $1 \mathrm{~A}$ ). A significantly decreased bone volume (BV) and BMD was further observable in female animals compared to their male counterparts after transplantation of collagen type I fibers (Fig. 1B) or Spongostan (Fig. 1C). In particular, a complete closure of the lesion was observable in Spongostantreated male rats, while female rats revealed only partial recovery (Fig. 1C). Histological examination of the newly formed bone confirmed the observations made via $\mu \mathrm{CT}$, particularly revealing increased amounts of new formed bone in male rats compared to female animals after transplantation of Spongostan or collagen type I fibers (Fig. 1 D, stars indicate new bone). Thus, we demonstrate here a strong and sex-specific impairment of female rats in regenerating calvarial bone lesions compared to males.

\section{Female adult human craniofacial stem cells show strongly delayed osteogenic differentiation}

Next to assessing sex-specific differences in bone regeneration of calvarial lesions in vivo, we investigated potential sex-dependent differences in osteogenic differentiation in a human model system. We used human craniofacial NCSCs from both sexes and cultivated them on collagen fibers (Fig. 3A) to induce osteogenic differentiation by physical cues only (16). NCSCs from male individuals revealed a strong Alizarin Red S-stained calcium deposition indicating successful osteogenic differentiation after 30 days of culture (Fig. 3B). On the contrary, we observed no signs of Alizarin Red S-positive calcification in female NCSCs cultivated under the same conditions for 30 days (Fig. 3C). After 40 additional days (day 70) of culture on collagen type I fibers, NCSCs from female donors were also able to differentiate and showed Alizarin Red S-stained calcium deposits (Fig. S1). In summary, adult NCSCs from female individuals differentiated on collagen type I fibers but revealed a strong delay in osteogenic differentiation over time in comparison to male NCSCs (under physiological differentiation cues without hormones). 

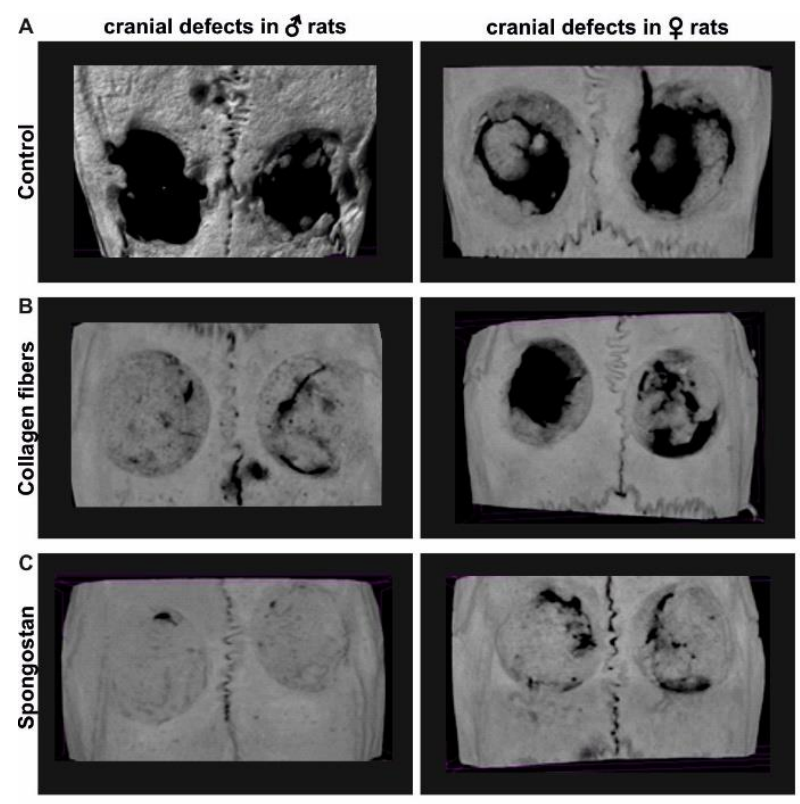

D
혼
ㅎํㄴ
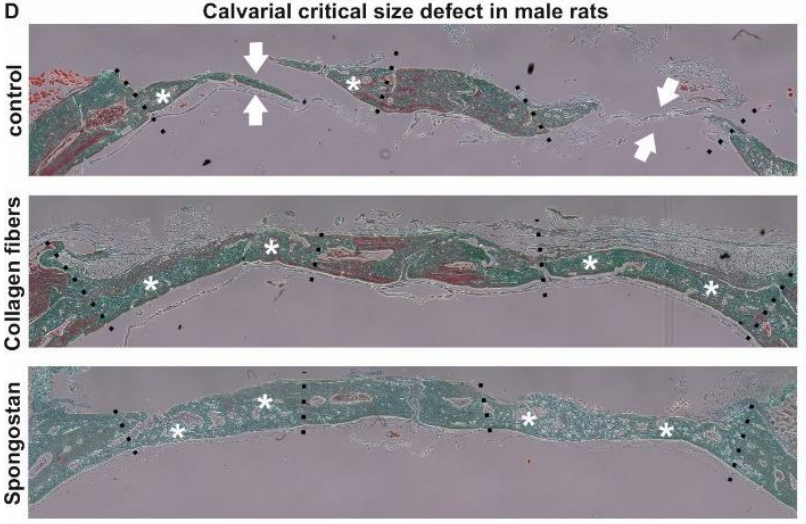
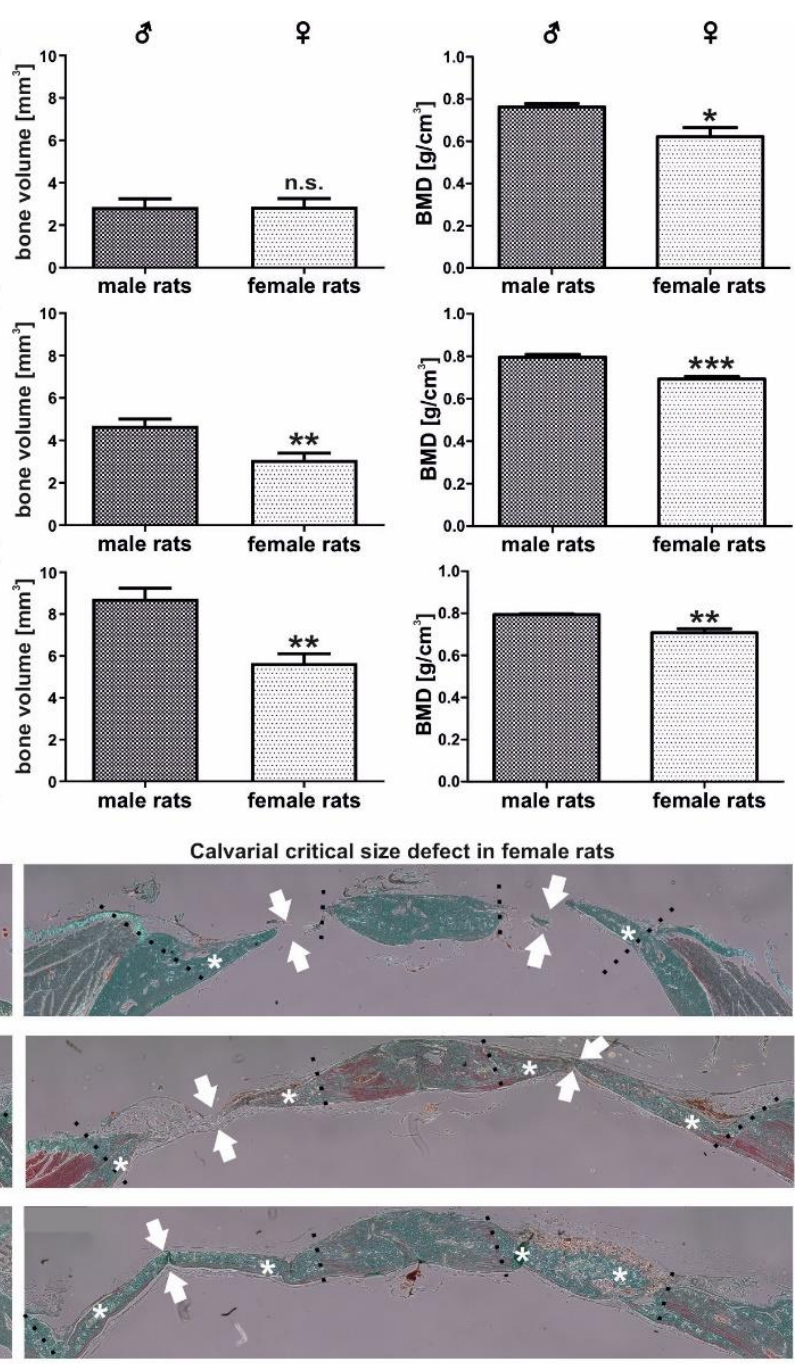

Figure 1. Female rats show a reduced capacity for regenerating calvarial bone lesions after transplantation of Spongostan or collagen type I fibers into critical-size calvarial defects. (A) Micro-computed tomography $(\mu \mathrm{CT})$ followed by quantification of bone volume and bone mineral density (BMD) revealed no closure of the criticalsize defects in female or control male animals, but a significantly decreased BMD in female control rats. Mann Whitney test, ${ }^{*} \mathrm{P}<0.05,{ }^{* *} \mathrm{P}<0.01,{ }^{* * *} \mathrm{P}<0.001$ was considered significant. (B-C) $\mu \mathrm{CT}$ scans depicting a significantly decreased bone volume and BMD in female animals compared to their male counterparts after transplantation of collagen fibers or Spongostan. (D) Histological examination revealed elevated levels of newly formed bone in male animals upon transplantation of collagen fibers or Spongostan in male rats. Stars mark newly formed bone, arrows indicate unclosed lesion. 
bioRxiv preprint doi: https://doi.org/10.1101/2021.11.10.468047; this version posted November 11, 2021. The copyright holder for this preprint (which was not certified by peer review) is the author/funder, who has granted bioRxiv a license to display the preprint in perpetuity. It is made available under aCC-BY-NC-ND 4.0 International license.
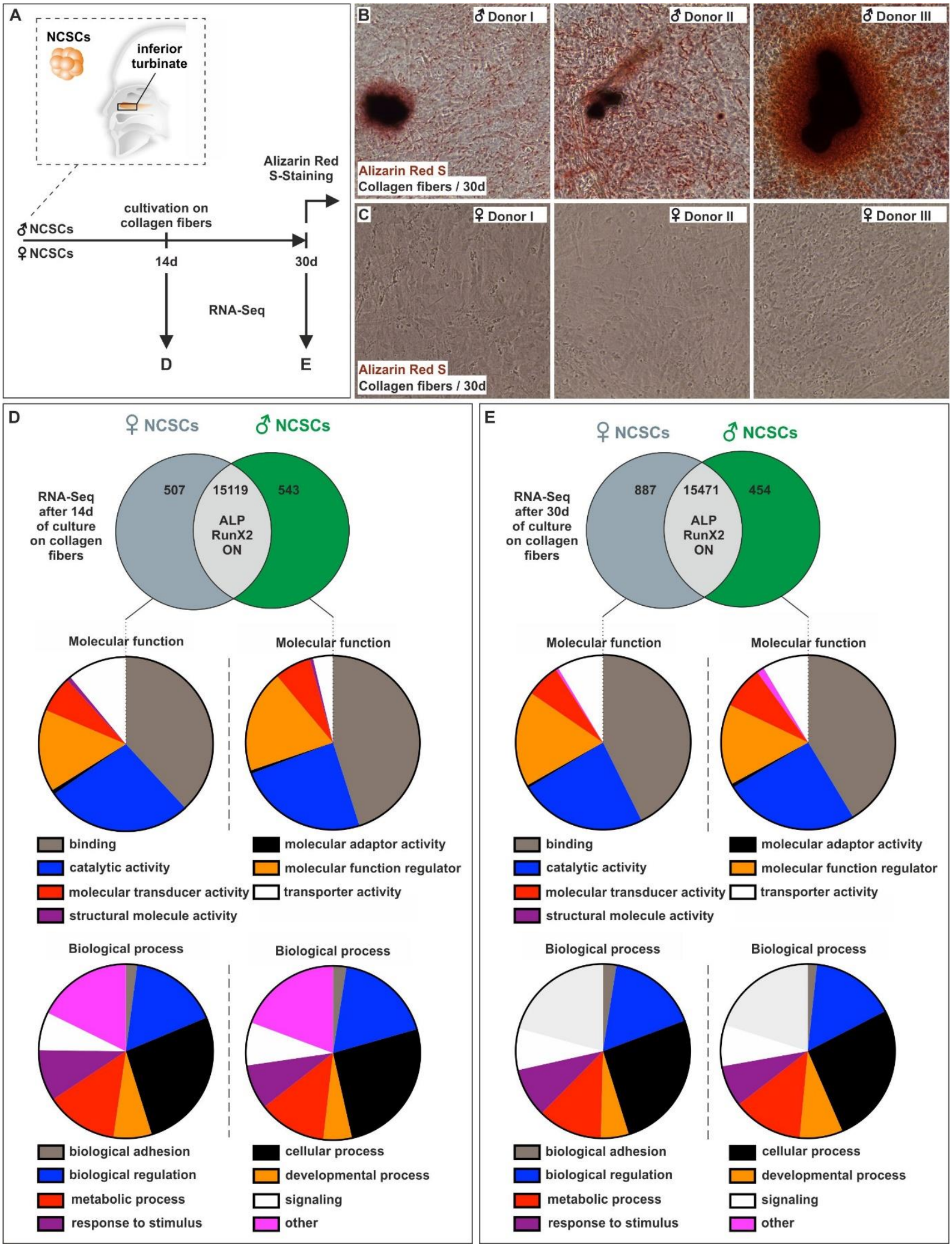

129 Figure 2. Adult human neural crest-derived stem cells from female donors show a strong delay in osteogenic differentiation in comparison to male NCSCs. (A) Schematic depiction of the endogenous niche of craniofacial NCSCs as well as the experimental design. Scheme partly modified from (16) (B-C) NCSCs from male individuals showed a strong Alizarin Red S-stained calcium deposition after culture on collagen fibers for 30 days, while no signs of Alizarin Red S-positive calcification were observable in female NCSCs. (D-E) RNA-sequencing revealed the 
expression of common osteogenic markers like ALP, Runx2 and Osteonectin in male and female NCSCs after 14 and 30 days, while transcripts solely expressed in either male or female NCSCs belonged to similar GO-terms.

\section{Transcriptome analysis reveals an extreme sexually dimorphic gene expression}

To determine the molecular basis of their sexually dimorphic differentiation behavior, RNA-sequencing of adult human craniofacial NCSCs was performed after 14 and 30 days of osteogenic differentiation. Global gene expression profiling revealed the expression of already known osteogenic markers like ALP, Runx2 and Osteonectin in male and female NCSCs after 14 and 30 days of differentiation without observable differences between the sexes (Fig. 2D-E). We found 507 (14 days) and 887 (30 days) transcripts solely expressed in male NCSCs, while 543 (14 days) and 454 (30 days) were exclusively present in female NCSCs upon differentiation (Fig. 2D-E). Interestingly, we observed strong differences in the percentages of genes belonging to the molecular function GO-terms "binding" and "molecular function regulator", which were elevated in male NCSCs compared to female ones after 14 days of differentiation (Fig. 2D). On the contrary, the percentages of genes belonging to the molecular function GO-term "transporter activity" was found to be increased in female NCSCs (Fig. 2D). We further observed nearly similar amounts of molecular function-related genes after 30 days of differentiation between male and female NCSCs (Fig. 2E). Likewise, female and male NCSCs revealed nearly similar percentages of expressed genes belonging to biological process GO terms like "biological adhesion", "biological regulation", "cellular process", or "response to stimulus" (Fig. 2D-E). These findings show that female NCSCs at least to some extend undergo osteogenic differentiation upon culture on collagen fibers despite the lack of calcium deposition after 30 days.

On the contrary, in male NCSCs differential gene expression analysis revealed a significantly elevated expression of characteristic osteogenic markers and regulators like BMP4, MSX2, C-FOS, COL4A4, COL11A1 and ITGA1 (table 1). In addition to this osteogenic expression pattern, hierarchical clustering of differentially expressed genes (DEGs) showed a cluster of upregulated genes in male NCSCs comprising amongst others the known autosomal osteogenic regulator Cytochrome P450 Family 7 Subfamily B Member 1 (CYP7B1) and the InRNA family with sequence similarity 157 (FAM157C) (Fig. 3A-B). Amongst other, this cluster further included Y-linked genes like the lysin demethylase 5D (KDM5D) and the putative osteogenic regulator Ubiquitin Specific Peptidase 9 Y-Linked (USP9Y) (Fig. 3A-B). Contrarily, female NCSCs revealed an increased expression of BMP7 (table 1) as well as a cluster of upregulated genes comprising the pro-osteogenic regulators KDM6A and XIST compared to male after 30 days of differentiation (Fig. 3C-D). However, we observed negative regulators of osteogenic differentiation like the X-linked gene taxilin gamma (TXLNG) (Fig. 3C-D) or MIR137HG (table 1) to be significantly higher expressed in female NCSCs in comparison to their male counterparts. 
bioRxiv preprint doi: https://doi.org/10.1101/2021.11.10.468047; this version posted November 11, 2021. The copyright holder for this preprint (which was not certified by peer review) is the author/funder, who has granted bioRxiv a license to display the preprint in perpetuity. It is made available under aCC-BY-NC-ND 4.0 International license.

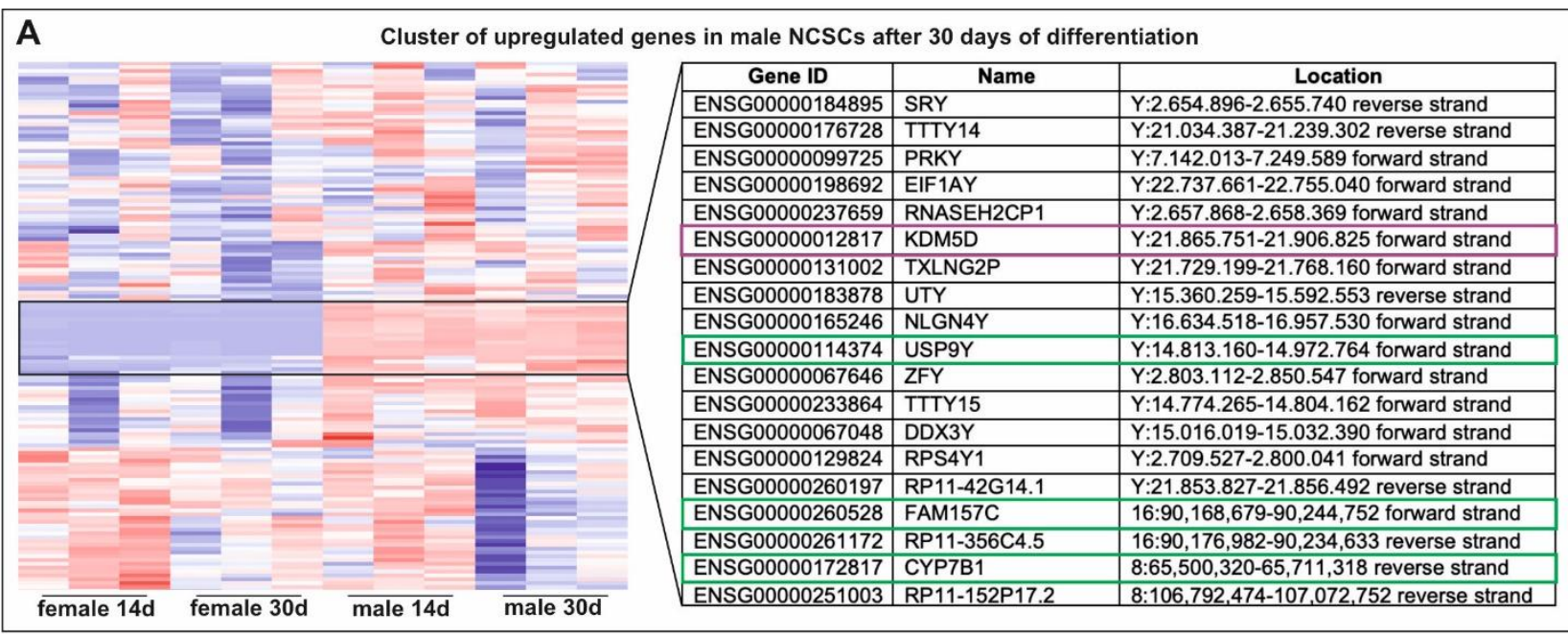

B

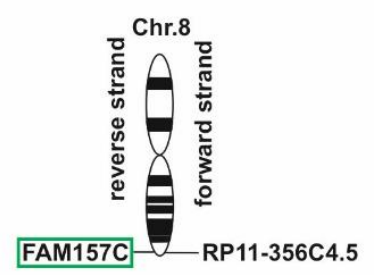

Chromosomal location of upregulated genes in Cluster $\mathrm{A}$
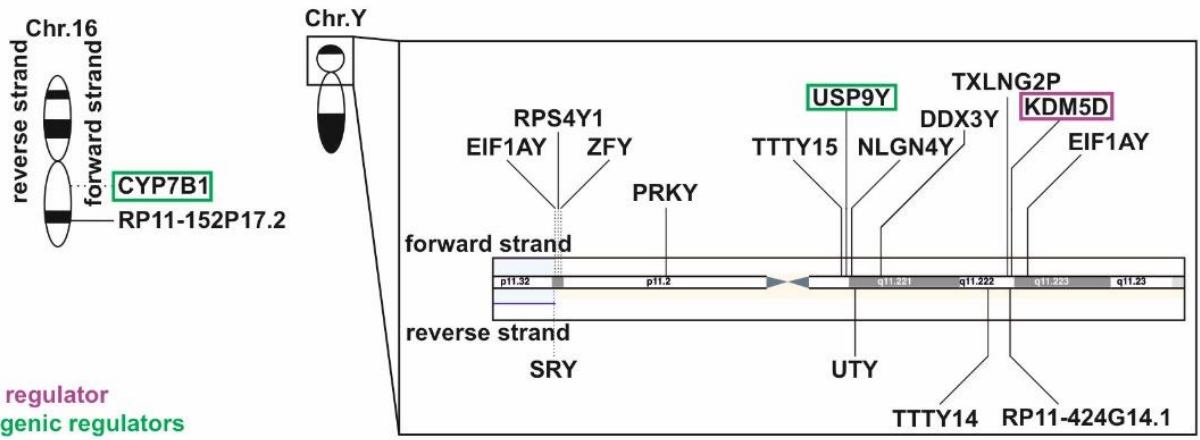

Novel putative positive osteogenic regulator

Known and putative positive osteogenic regulators

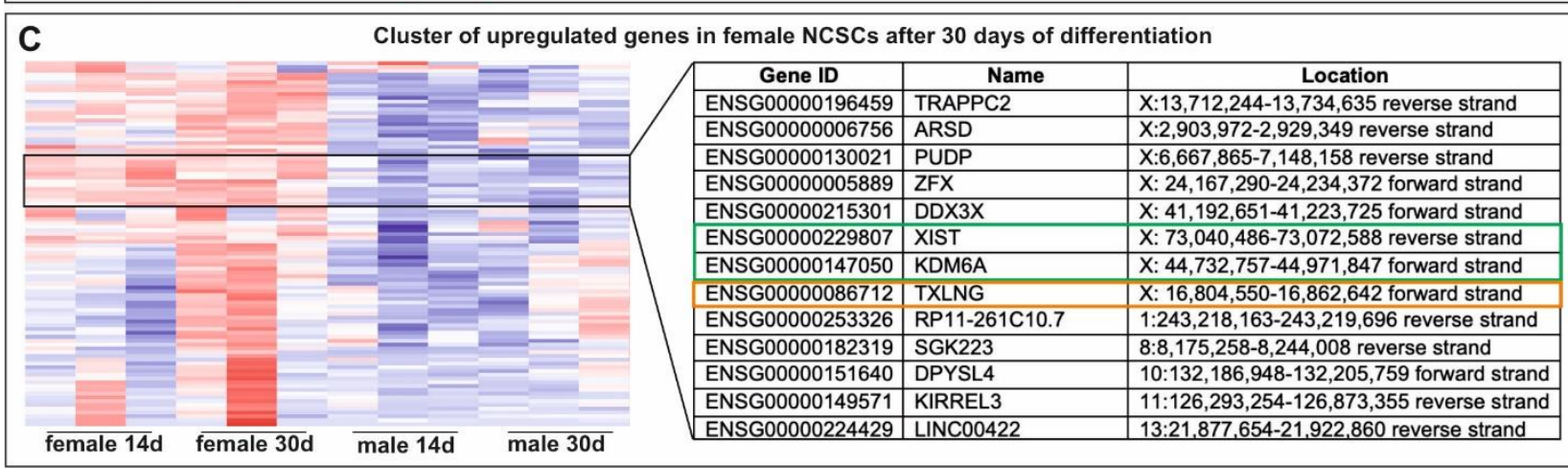

D

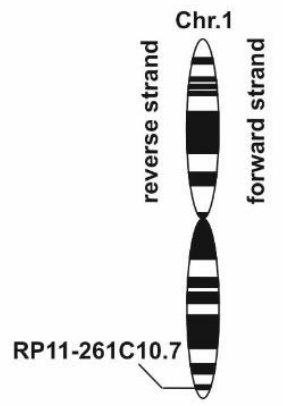

Chromosomal location of upregulated genes in Cluster C
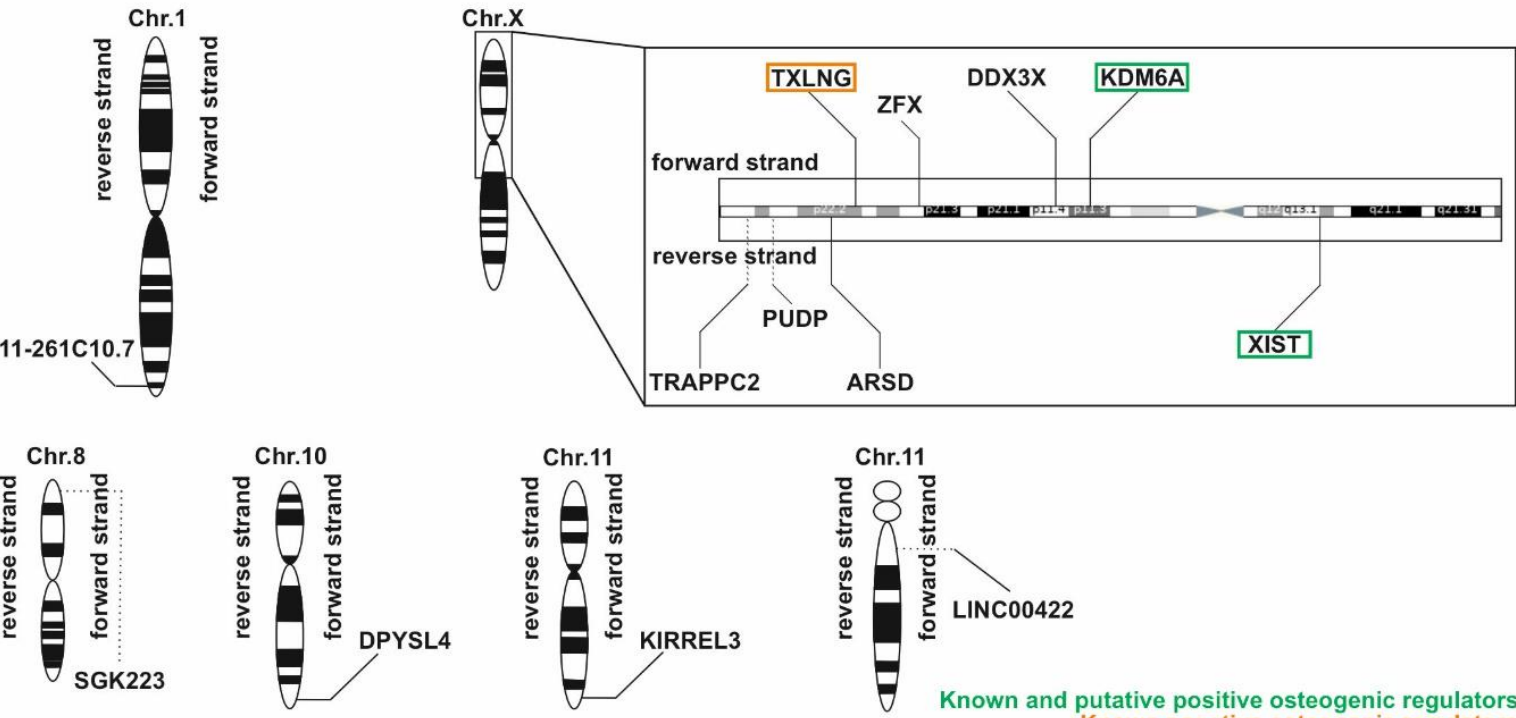

d putative positive osteogenic regulators Known negative osteogenic regulators 
Figure 3. Hierarchical clustering of differentially expressed genes reveal a sexually dimorphic osteogenic regulator profile between male and female NCSCs undergoing differentiation. (A-B) Cluster of upregulated genes in male NCSCs comprising autosomal osteogenic regulator CYP7B1 as well as the Y-linked genes KDM5D and USP9Y. (C-D). Female NCSCS showed cluster of upregulated genes including the pro-osteogenic regulators KDM6A and XIST as well as the negative regulator TXLNG.

Table 1: Differentially expressed pro- and anti-osteogenic genes not included in clusters (Figure 3) between female and male NCSCs after 30 days of differentiation.

\begin{tabular}{|c|c|c|c|}
\hline Gene name & ENS ID & $\begin{array}{c}\text { Down-/upregulation in female } \\
\text { NCSCs compared to male NCSCs } \\
\text { (log2 fold change) }\end{array}$ & P-valule \\
\hline BMP4 & ENSG00000125378 & -1.2 & 0.014 \\
\hline MSX2 & ENSG00000120149 & -0.8 & 0.013 \\
\hline COL11A1 & ENSG00000060718 & -2.8 & 0.015 \\
\hline COL4A4 & ENSG000000081052 & -1.2 & 0.043 \\
\hline ITGA1 & ENSG00000213949 & -0.8 & 0.021 \\
\hline FOS & ENSG00000170345 & -1.5 & 0.000 \\
\hline BMP7 & ENSG00000101144 & 2.6 & 0.008 \\
\hline MIR137HG & ENSG00000225206 & 1.0 & 0.037 \\
\hline
\end{tabular}

179 In addition to the determination of distinct clusters of differentially expressed genes or single 180 osteogenic markers and regulators, we performed Kyoto Encyclopedia of Genes and Genomes (KEGG) pathway and Gene Ontology (GO) term analysis. In accordance to our findings showing differences in the osteogenic differentiation between male and female NCSCs, differentially expressed genes belonged amongst others to the osteogenic-related KEGG pathways "ECM-receptor-interaction", "cell adhesion molecules", "focal adhesion", "TGF-beta signaling pathway" or "MAPK signaling pathway" (Fig. 4C). Accordingly, differentially expressed genes were annotated to the GO terms "cell adhesion", "cytoskeleton organization" or "extracellular region" (Fig. 4C). 
bioRxiv preprint doi: https://doi.org/10.1101/2021.11.10.468047; this version posted November 11, 2021. The copyright holder for this preprint (which was not certified by peer review) is the author/funder, who has granted bioRxiv a license to display the preprint in perpetuity. It is made available under aCC-BY-NC-ND 4.0 International license.
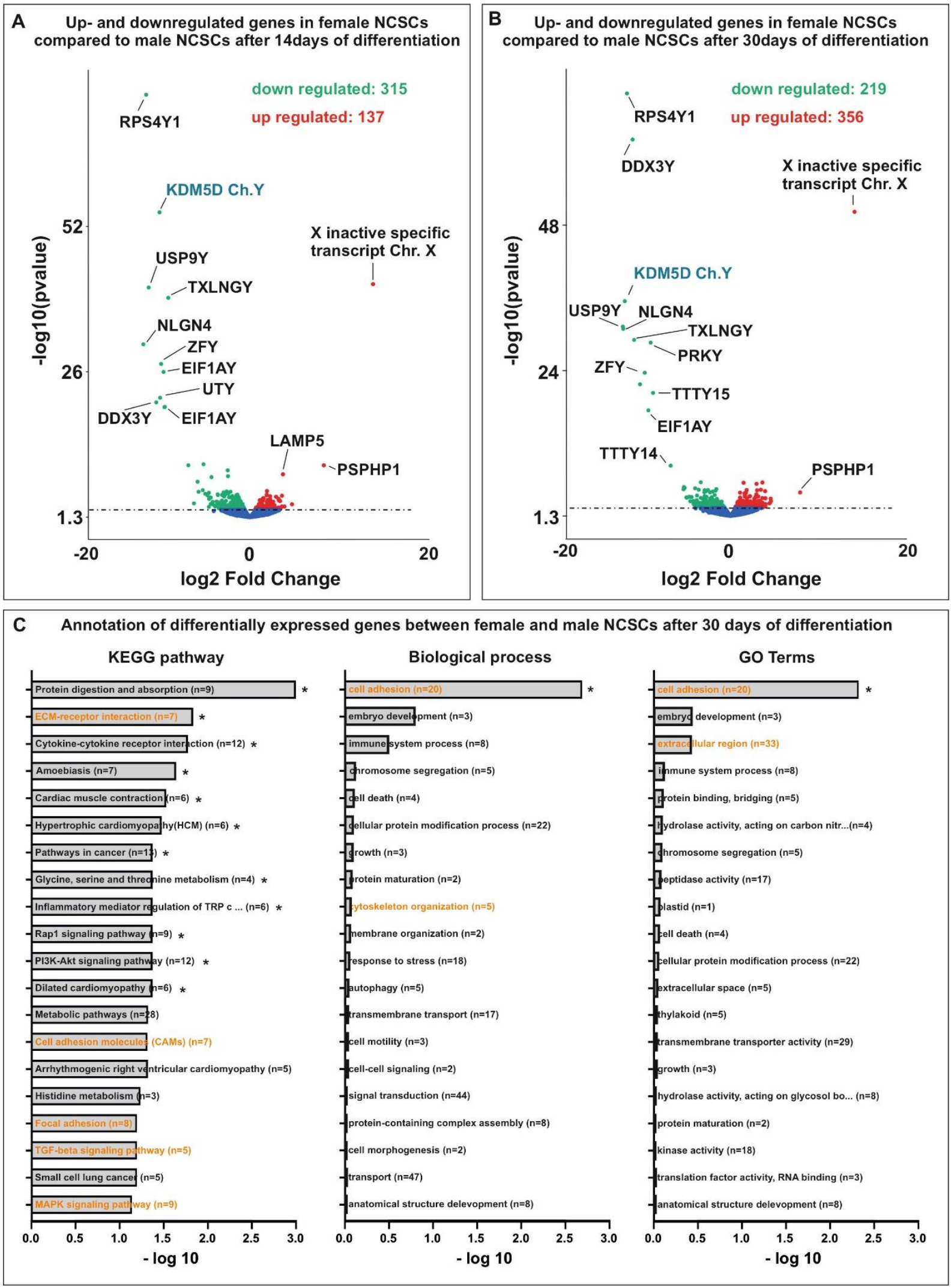

190 Figure 4. (A-B) Ribosomal Protein S4 Y-Linked 1 (RPS4Y1) and KDM5D (depicted in in cyan) were most significantly downregulated in female NCSCS after 14 days and 30 of differentiation compared their male counterparts. (C) Differentially expressed genes between male and female NCSCs belonged amongst others to the osteogenicrelated KEGG pathways and GO terms (depicted in orange). 
Identification of Y-linked lysin demethylase $5 \mathrm{D}$ as a novel regulator of osteogenic differentiation in male NCSCs

Among the genes in cluster A (Fig. 3A-B) upregulated in male NCSCs undergoing osteogenic differentiation, we found Ribosomal Protein S4 Y-Linked 1 (RPS4Y1) and lysin demethylase 5D (KDM5D) to be most significantly und strongly downregulated in female NCSCS after 14 days of differentiation (Fig 4A). In addition to DEAD-Box Helicase 3 Y-Linked (DDX3Y), the transcripts of RPS4Y1 and KDM5D were likewise most significantly downregulated in female NCSCs undergoing osteogenic differentiation for 30 days (Fig 4B). While increased transcription of RPS4Y1 might be a potential hint of differences in protein translation, histone methylation and histone demethylases like KDM4A are discussed to regulate osteogenic differentiation (reviewed in (20))(21). Thus, we particularly focused on investigating the regulatory functions of KDM5D in more detail. We used siRNAs against five distinct loci in the mRNA of KDM5D (Fig. 5A) followed by their lentiviral transduction into male NCSCs. Firstly, knockdown of KDM5D was verified by qPCR depicting a significantly reduced expression of KDM5D compared to untreated control and GFP-transduced control in undifferentiated NCSCs (Fig. 5B). Secondly, knockdown of KDM5D resulted in a strong inhibition of osteogenic differentiation of male NCSCs shown by reduced Alizarin Red S-stained calcium deposition after 30 days of culture on collagen type I fibers (Fig. 5C). Quantification of Alizarin Red S-stainings confirmed the significant inhibition of male osteogenic differentiation upon siRNA-mediated knockdown of KDM5D (Fig. 5D-E). In addition, we applied KDOAM-25 for pharmacological inhibition of KDM5D in male NCSCs. After 30 days of culture on collagen type I fibers, we observed a significant reduction of calcium deposition in male NCSCs with increasing concentrations of KDOAM-25 $(1 \mu \mathrm{M}-100 \mu \mathrm{M})$ compared to untreated control differentiation in male craniofacial NCSCs. 

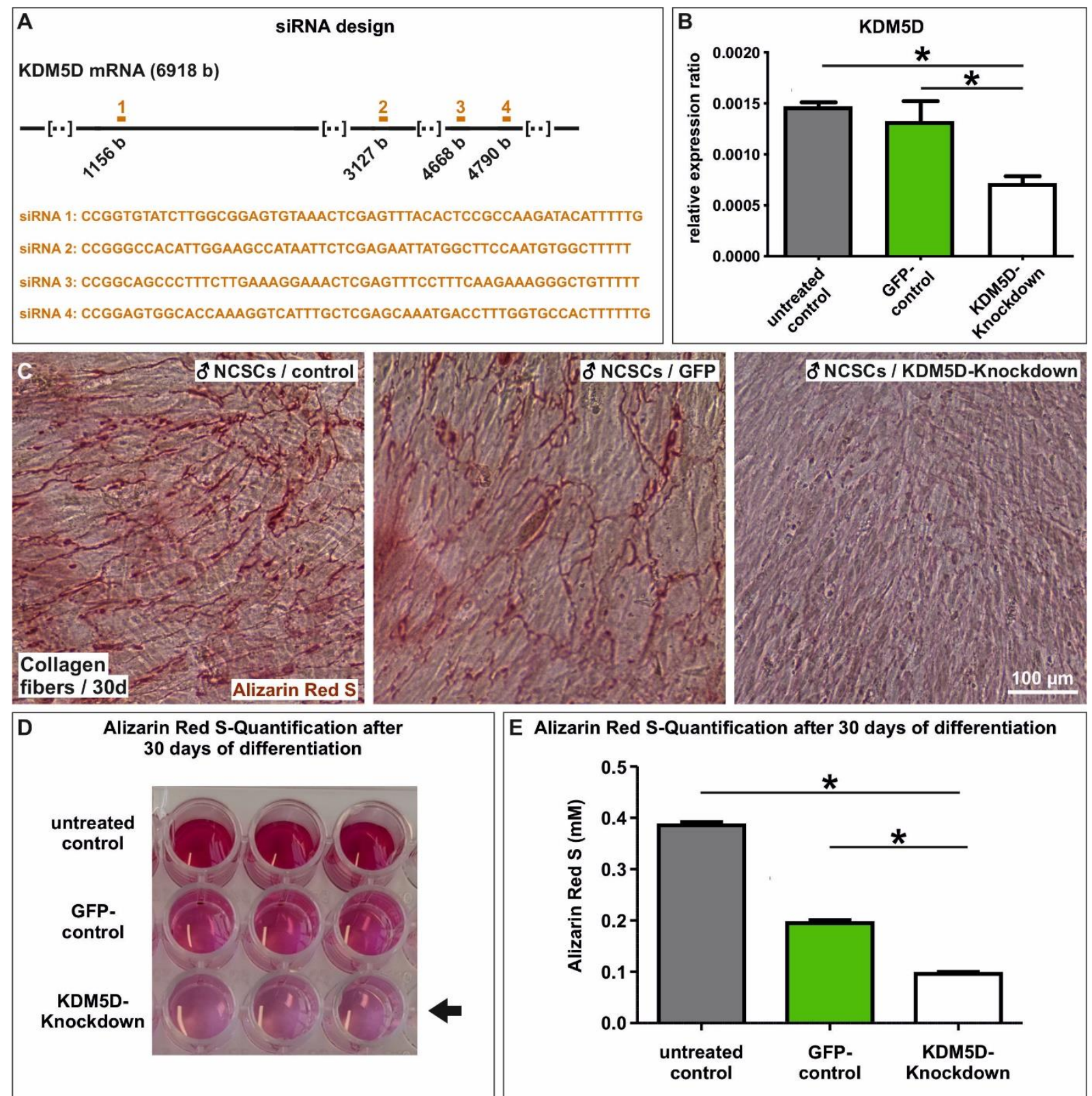

E Alizarin Red S-Quantification after 30 days of differentiation

Figure 5. Knockdown of KDM5 significantly impairs the osteogenic differentiation capacity of male NCSCs. (A)

Schematic view on siRNA design. (C) qPCR analysis validating the knockdown of KDM5D. (C-E) Knockdown of KDM5D strongly inhibited the capability of male NCSCs for depositing calcium after 30 days of culture on collagen type I fibers compared to untreated and GFP-transduced cells. (D-E) Quantification of Alizarin Red S confirmed the significant inhibition of osteogenic differentiation in male NCSCs after knockdown of KDM5D. Mann Whitney test, $* \mathrm{P}<0.05$ was considered significant. 

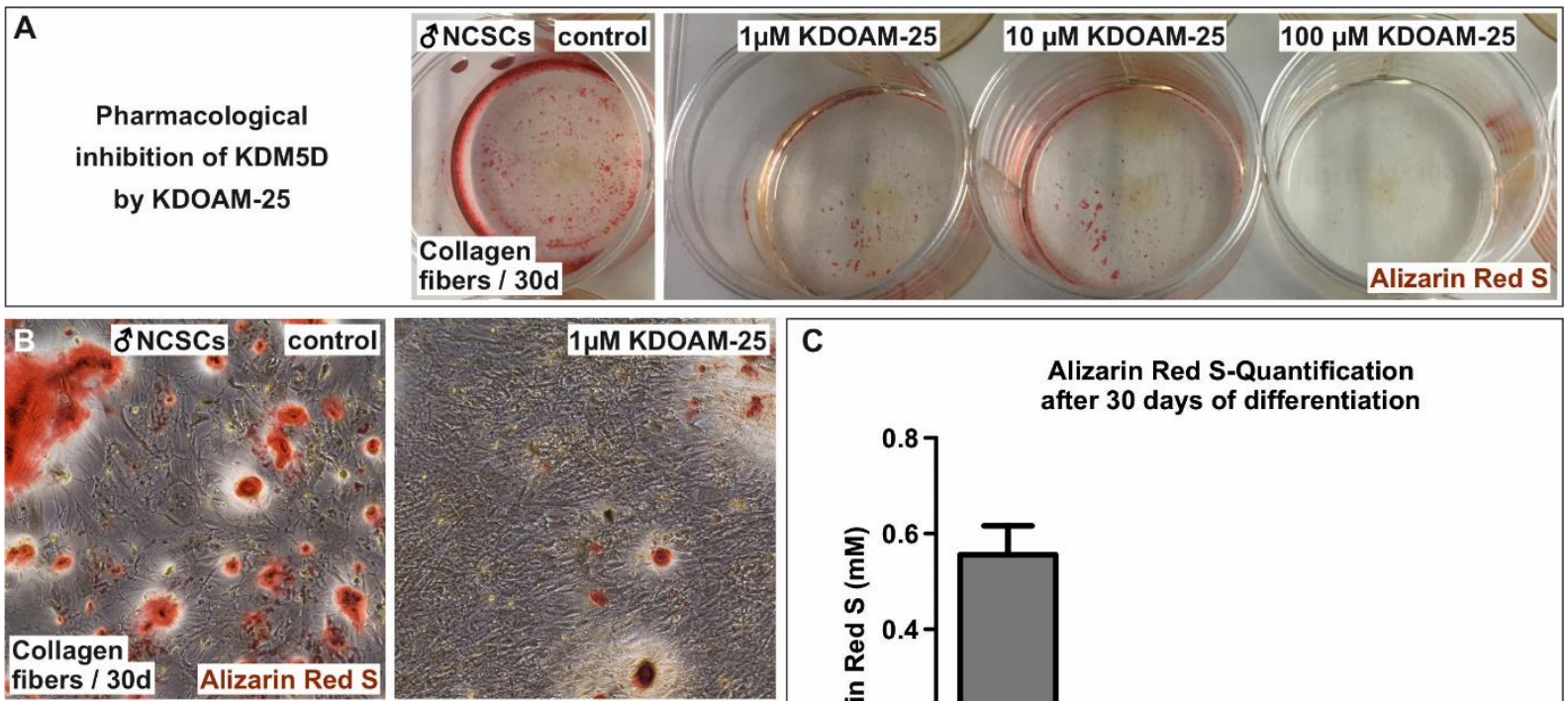

$1 \mu \mathrm{M} \mathrm{KDOAM}-25$
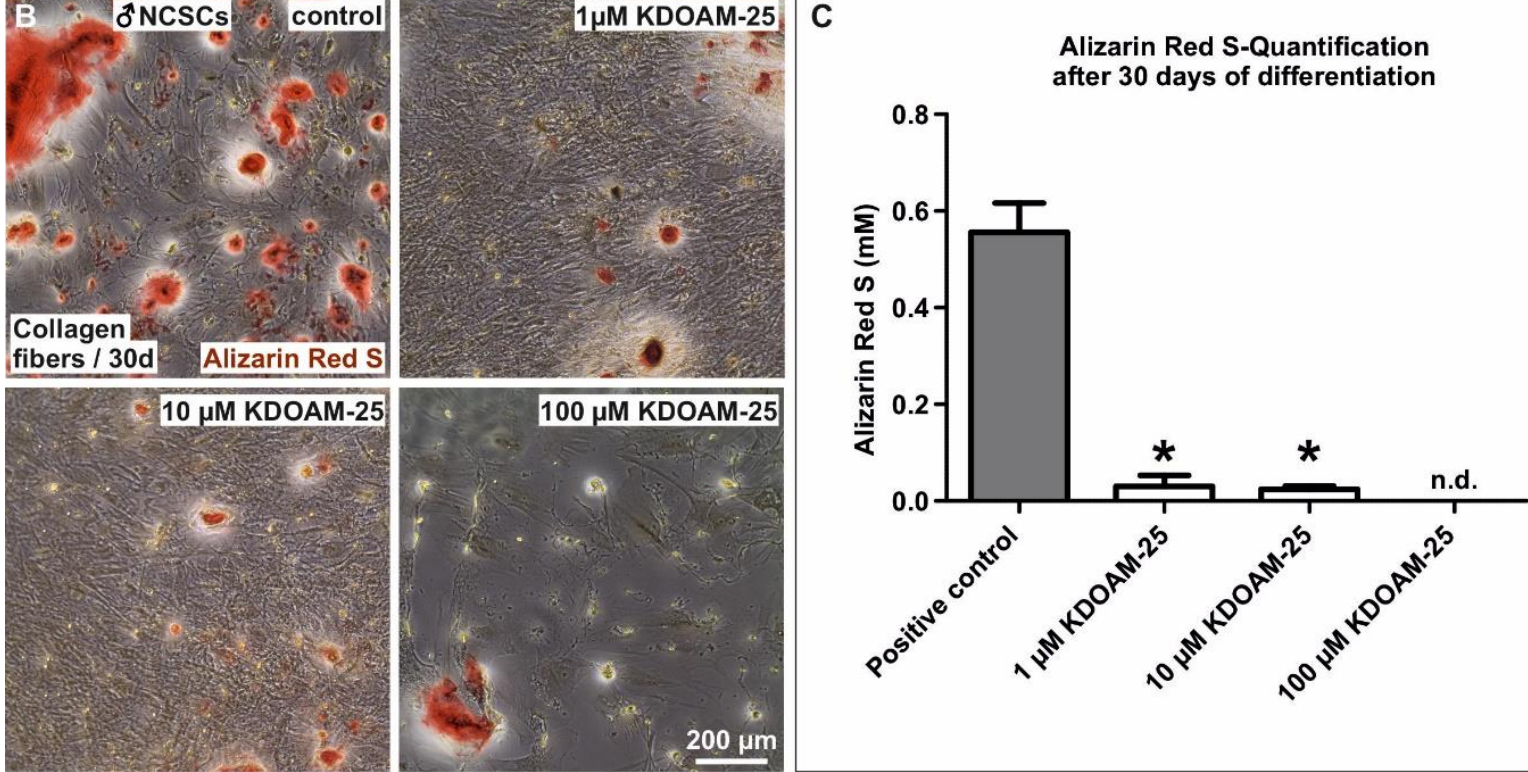

Figure 6. Pharmacological inhibition of KDM5D significantly reduces the capability of male NCSCs to undergo osteogenic differentiation. (A-B) Exposure of male NCSCs to increasing concentrations $(1 \mu \mathrm{M}-100 \mu \mathrm{M})$ of KDOAM-25 resulted in a significant reduction of calcium deposition after 30 days of differentiation compared to untreated control. (C) Quantification of Alizarin Red S confirmed the significant inhibition of male osteogenic differentiation upon inhibition of KDM5D by KDOAM-25-treatment. Mann Whitney test, ${ }^{*} \mathrm{P}<0.05$ was considered significant. n.d.: not detectable. 


\section{Discussion}

In the present study, we demonstrate a decreased capacity of calvarial bone recovery in female rats as well as a cell autonomous delay in osteogenic differentiation of female human craniofacial stem cells compared to their male counterparts. Our findings particularly reveal a significantly decreased bone volume and bone mineral density in female rats compared to male animals upon transplantation of Collagen fibers or Spongostan into critical-size calvarial defects. Accordingly, we previously reported the highly efficient closure of the calvarial lesions in male rats by Collagen fibers or Spongostan (18) even in comparison to other clinically approved bone substitute materials like NanoBone or Actifuse (19). In line with our present observations, Meszaros and coworkers reported a significantly increased regeneration of cranial bone defects in male mice compared to their female counterparts after transplantation of gelatin sponges containing muscle-derived stem cells (12).

Transferring these promising findings to the human system, we further observed a strong delay in osteogenic differentiation of adult human craniofacial neural crest-derived stem cells from female donors compared to those isolated from male ones. This observation is in accordance with the reduced capacity of female individuals for bone regeneration (1), reviewed in $(2,3,4)$ and increased risk of fractures developing nonunion $(1,6)$ and elevated prevalence of osteoporosis (7-9). Although certain osteogenic markers like Runx2 or Osteonectin were not differentially expressed between male and female NCSCs, global transcriptional profiling revealed a broad range of differentially expressed genes between male and female NCSCs after 30 days of differentiation. Amongst others, these genes belonged to the KEGG pathway "ECM-receptor-interaction", "cell adhesion molecules", "focal adhesion", "TGF-beta signaling pathway", and "MAPK signaling pathway" or the GO term "cell adhesion". In line with our present findings, KEGG pathways like "focal adhesion", "ECM-receptor interaction" and the "MAPK signaling pathway" were previously reported to be enriched in human adult stem cells undergoing osteogenic differentiation compared to undifferentiated controls (Shang et al. 2016). Particularly the pathway "ECM-receptor interaction" and expression of the gene ITGA1 can be directly linked to an interaction of NCSCs with the substrate consisting of collagen type I fibers harboring a nanoporous surface of $30 \mathrm{~nm}$ pores, in turn guiding their osteogenic differentiation (16). Next to characteristic osteogenic markers like BMP4 $(22,23), \operatorname{MSX2}(24), \operatorname{COL} 4 A 4$ and COL11A1 (25), we observed a range of osteogenic regulators strongly upregulated in male NCSCs after osteogenic differentiation compared to their female counterparts. Notably, we found autosomal genes like C-FOS (chromosome 14), CYP7B1 (Cytochrome P450 Family 7 Subfamily B Member 1, chromosome 16) and FAM157C (family with sequence similarity 157, chromosome 8) among these regulators. Although CFOS was described to determine the differentiation of murine hematopoietic cells into osteoclasts (26), it was likewise shown to precede osteogenic differentiation of cartilage cells (27). Likewise, Ohta and 
colleagues reported fracture healing to be accompanied by an induction of C-FOS expression in osteoblasts within the ossifying callus (28). Next to C-FOS, our present data revealed an elevated expression of CYP7B1 in male NCSCs after 30 days of osteogenic differentiation. CYP7B1 encodes a P450 enzyme, namely the oxysterol $7 \alpha$-hydroxylase, which metabolizes the cholesterol metabolite 27hydroxycholesterol $(27 \mathrm{HC})$, the first identified endogenous selective estrogen receptor modulator (reviewed in (29)). In patients, CYP7B1 mutations consequently lead to an accumulation of circulating $27 \mathrm{HC}(30)$, which was in turn reported to inhibit the effects of estrogen (31). Notably, CYP7B1 can be also considered as a major regulator of osteogenesis, as DuSell and coworkers demonstrated a KO of Cyp7b1 in female mice to result in decreased bone mineral density and bone formation rate compared to wildtype controls (32). Likewise, we detected a male-specific elevated expression of the IncRNA FAM157C, which is commonly known to be expressed in the human bone marrow (33). Li and coworkers showed deletion of FAM157C as one rare copy number variant being present in female patients suffering from turner syndrome (34). Turner syndrome is a congenital disease caused by loss of one X-chromosome, which results amongst other phenotypic characteristics in a severe bone fragility including low bone mineral density and an elevated fracture risk (reviewed in (35)). In this line, the present observations reveal FAM157C as well as CYP7B1 and C-FOS as autosomal regulatory hallmarks of the osteogenic differentiation of male adult human stem cells compared to their female counterparts. Our findings may suggest the reduced expression of these regulators in differentiating female NCSCs as one underlying mechanism for the delay in osteogenic differentiation observed here. Next to autosomal osteogenic regulators, the expression of genes like USP9Y located on the $Y$ chromosome was significantly increased in male NCSCs compared to female NCSCs undergoing osteogenic differentiation. Interestingly, Shang and coworkers reported USP9Y to be alternatively spliced upon osteogenic differentiation (36), suggesting a regulatory role in osteogenic differentiation. We further particularly focused on one member of the Y-linked Lysin demethylase (KDM) family, namely KDM5D, which was highly upregulated in male NCSCs after 30 days of osteogenic differentiation compared to female NCSCs. Histone methylation is commonly known to be crucially involved in osteogenic differentiation of stem cells, although the methylation status can either lead to suppression or promotion of osteogenic differentiation (reviewed in (20)). For instance, overexpression of KDM4A specific for H3K9me3 (trimethylated Lysin-9 at histone H3) enabled osteoblast differentiation of mesenchymal stem cells (21). Likewise, Yin and coworkers demonstrated the histone H3K4 trimethyltransferase Ash1l (absent, small, or homeotic-like) to promote osteogenic differentiation and osteogenesis (37). On the contrary, KDM7A and KDM5A were reported to negatively regulate osteogenic differentiation of mesenchymal progenitor cells (KDM7A) or human adipose-derived stem cells (KDM5A) $(38,39)$. To the best of our knowledge, our present findings identify for the first time KDM5D, which demethylates H3K4me2/H3K4me1, as a critical driver of male 
osteogenic differentiation. Next to its elevated expression in male NCSCs after osteogenic differentiation, si-RNA-mediated knockdown or pharmacological inhibition of KDM5D significantly reduced the osteogenic differentiation capacity of male NCSCs. Therefore, our findings demonstrate the expression of KDM5D as prerequisite for the faster osteogenic differentiation of male NCSCs in comparison to NCSCs from female individuals. In accordance to our findings, Shang and coworkers reported KDM5D to be alternatively spliced in human cartilage endplate-derived stem cells after osteogenic differentiation compared to control (Shang et al. 2016).

While certain osteogenic regulators like KDM5D, C-FOS, CYP7B1 and FAM157C were highly upregulated in male NCSCS after osteogenic differentiation, we observed a completely different expression pattern of osteogenic regulators in female NCSCs after the same exposure period to differentiation stimuli. Here, we found elevated transcript levels of X-linked pro-osteogenic genes like KDM6A or XIST to be upregulated in female NCSCs compared to male ones. Hemming and coworkers reported the lysin demethylase KDM6A to promote osteogenic differentiation of MSCs in vitro and in vivo (40). The long non-coding RNA (InRNA) XIST was likewise shown to participate in osteogenic differentiation of NC-derived periodontal ligament stem cells (41). In line with the unchanged expression of osteogenic markers like Osteonectin and Runx2 between male and female NCSCs, these findings indicate the at least partial osteogenic differentiation of female NCSCs after 30 days of cultivation on collagen type I fibers. However, we also observed negative regulators of osteogenic differentiation to be significantly higher expressed in female NCSCs. These regulators particularly included the autosomally located gene MIR137HG (chromosome 1) as well as the X-linked gene TXLNG. Knockdown of MIR137HG was reported to promote the osteogenic differentiation of human adiposederived stem cells (42). The TXLNG gene encodes FIAT (factor inhibiting activating transcription factor 4 (ATF4)-mediated transcription), which overexpression in mice was shown to lead to a reduced mineral deposition rate in turn resulting in decreased bone mineral density and bone volume (43). These observations are in line with our present observations linking an elevated expression of TXLNG to a delayed osteogenic differentiation and calcium deposition in female NCSCs. The expression of these negative osteogenic regulators in female NCSCs in combination with the decreased expression of major positive regulators like KDM5D, C-FOS or CYP7B1 compared to their male counterparts may build the molecular basis of the observed delay in osteogenic differentiation in female craniofacial NCSCs.

344 In summary, our present findings show a decreased calvarial bone regeneration in female rats as well as a delayed osteogenic differentiation of female adult stem cells from the craniofacial region compared to their male counterparts. On molecular level, this delay in osteogenic differentiation was accompanied by sex-specific expression pattern of negative osteogenic regulators like MIR137HG and

348 TXLNG in female NCSCs, while male NCSCs revealed an elevated expression of pro-osteogenic 
bioRxiv preprint doi: https://doi org/10.1101/2021.11.10.468047; this version posted November 11,2021 . The copyright holder for this preprint (which was not certified by peer review) is the author/funder, who has granted bioRxiv a license to display the preprint in perpetuity. It is made available under aCC-BY-NC-ND 4.0 International license.

349 regulators including C-FOS and CYP7B1. We further demonstrate the expression of Y-linked KDM5D as

350 a prerequisite for the faster osteogenic differentiation of male NCSCs in comparison to NCSCs from

351 female individuals. The present observation highlight the relevance of sexual dimorphisms in stem cell

352 behavior for guiding bone recovery in clinical settings.

353

354

355 


\section{Materials and Methods}

357

358

359

360

361

362

363

\section{Animals}

This study was approved by the responsible authority (LANUV, Approval Ref. No. 81-0204.2018.A188). All animal work was performed in accordance with the policies and procedures established by the Animal Welfare act, the National Institutes of Health Guide for Care and Use of Laboratory Animals, and the National Animal Welfare Guidelines. For this study, a minimum number of 5 male and 5 female white Wistar rats (average weight of $300 \mathrm{~g}$ at day of surgery) from our own breeding at the University of Bielefeld were randomly assigned to either group A (empty control, $n=5$ ), group $B$ (collagen fibers, $\mathrm{n}=5$ ), group C (Spongostan, $\mathrm{n}=5$ ). All animals were housed 2-per-cage under a $12 \mathrm{~h}$ light/dark cycle with food and water provided ad libitum. All efforts were made to minimize potential suffering in accordance to the $3 R$ (replacement, reduction, refinement) guidelines.

\section{Animal Surgery and Postoperative Care}

Male rats were anesthetized as previously described (18). Female rats were administered an intraperitoneal injection of "Hellabrunner Mischung" comprising ketamine hydrochloride/xylazine hydrochloride solution ( $1 \mathrm{ml} / \mathrm{kg}$, Sigma Aldrich, Taufkirchen, Germany). The surgical procedure followed the description of Spicer and colleagues (44). Briefly, the fur was shaved and the area of surgery was cleaned using an iodine swab. A skin incision, followed by periosteum removal, was made. Afterwards, on both sides the trepanation was performed using a $5 \mathrm{~mm}$ hollow drillhead (Trephines 229 RAL 040: Hager and Meisinger GmbH, Neuss, Germany) and a surgical dental drill (Implantmed: W\&H Dentalwerk Bürmoos GmbH, Bürmoos, Austria) with a constant speed of $2000 \mathrm{rpm}$. The circular bone fragment was lifted out, the defect was washed with sterile saline solution and the holes were treated according to the group the animal was assigned to. For void filling, Spongostan (Ferrosan Medical Devices, Søborg, Denmark; marketed by Ethicon Biosurgery, Johnson and Johnson, New Brunswick, NJ, United States) was commercially purchased, cut into cubic $\left(1 \mathrm{~mm}^{3}\right)$ and pre-wetted in $0.9 \% \mathrm{NaCl}$ solution (B. Braun Melsungen AG, Melsungen, Germany) for 30 min prior to transplantation (18). Collagen type I fibers were prepared as described previously (16). Thereafter, the periosteum was closed with sutures (Prolene 5-0, Ethicon, Johnson and Johnson, New Brunswick, NJ, USA) and the skin was closed by four single back-and-forth sutures (Prolene 3-0, Ethicon, Johnson and Johnson, New Brunswick, NJ, USA). After surgery, the rats were placed in a warmed incubator and housed singly for 3 days.

\section{Euthanasia and Sample Extraction}


Animals were euthanized thirty days postoperatively using the Exposure Line carbon dioxide box bioscope (Ehret, Freiburg, Germany). Calvaria were extracted by removing soft tissue and surrounding bone, followed by fixation using $4 \%$ paraformaldehyde (PFA) and storage at $4^{\circ} \mathrm{C}$.

\section{Micro-CT}

All samples underwent micro-computed tomography as previously described (18) using a SkySkan 1176 (Bruker, Kontich, Belgium).

\section{Histology}

Extracted calvaria were fixed with $4 \%$ paraformaldehyde (PFA), subsequently decalcified using ethylenediaminetetraacetic acid (EDTA) and embedded in paraffin. Serial sections were stained with Goldner's trichrome stain, while nuclei were stained with hematein followed by microscopical examination.

\section{Osteogenic Differentiation of Human Neural Crest Derived Inferior Turbinate Stem Cells}

Human inferior turbinates were obtained during routine surgery after an informed consent process, according to local and international guidelines (Bezirksregierung Detmold, Germany) and NCSCs (inferior turbinate stem cells, ITSCS) were isolated and cultivated as previously described (45). All experimental procedures were ethically approved by the ethics board of the medical faculty of the University of Münster (No. 2012-015-f-S). Osteogenic differentiation was induced topologically using collagen fibers according to our previously reported protocol (16). Briefly, cells were seeded onto collagen fibers endogenously harboring $30 \mathrm{~nm}$ pores and cultivated in DMEM supplemented with $10 \%$ fetal calf serum at $37^{\circ} \mathrm{C}$ and $5 \% \mathrm{CO}_{2}$ in a humidified incubator (Binder, Tuttlingen, Germany). Calcium deposition was visualized by Alizarin Red staining and imaging according to (16) followed by quantification using photometric measurement as shown in (46).

\section{RNA Isolation and Sequencing}

For RNA isolation the NucleoSpin RNA kit (Macherey-Nagel, Düren, Germany) was used according to manufacturer's guidelines. Quality and quantity of the isolated RNA was determined via NanoDrop (Thermo Fisher Scientific,Waltham, MA, USA). Library preparation using the NEB Next Ultra Library Prep Kit (New England Biolabs, Frankfurt am Main, Germany) and sequencing on Illumina Novaseq6000 platform was carried out by Novogene (Cambridge, United Kingdom) followed by bioinformatic 

qPCR above.

analysis. Hisat2 was used for mapping to the reference genome GRCh38/hg19. Quantification of the gene expression was analyzed via HTSeq and for differential gene expression analysis the DESeq2 R Package was used. Enrichment analysis of differentially expressed coding genes were determined for GO Enrichment via the R Packages GOSeq, topGO and hmmscan and analysis of Kyoto Encyclopedia of Genes and Genomes (KEGG) pathway enrichment was performed using KOBAS and PANTHER.

\section{Generation of lentiviral vectors}

ShRNAs against KDM5D were commercially purchased (MISSION shRNA Bacterial Glycerol Stock, clone IDs: NM_004653.3-4909s21c1, NM_004653.2-4787s1c1, NM_004653.3-1368s21c1, NM_004653.23246s1c1, Sigma Aldrich). Target sequences are depicted in Figure 5A. Lentiviral vectors were generated followed to the protocol of Tang and colleagues (47) using transient cotransfection of HEK 293T cells with a three-plasmid system. Lentiviral transfer vector plasmid, packaging plasmid and envelope plasmid were mixed and 1,25 mM PEI were added and incubated for $10 \mathrm{~min}$ at room temperature. Meanwhile, HEK 293 T cells were trypsinized, washed twice with $1 \times$ PBS and $2 \times 10^{6}$ cells were resuspended in media. The vector/PEI mixture was added to the cells and immediately plated onto a plate and incubated at $37^{\circ} \mathrm{C}$ and $5 \% \mathrm{CO}_{2}$ in a humidified incubator. After $48 \mathrm{~h}$ the supernatant containing lentiviral vectors was collected and centrifugated at 3000rpm for $5 \mathrm{~min}$ at room temperature followed by filter through a $0,45 \mu \mathrm{m}$ mesh. Virus was transferred into conical tubes and spin at 20000rpm for 2 hours at $4^{\circ} \mathrm{C}$ in (Beckman TST2838) swinging bucket rotor. After spin the supernatant was discarded and the virus resuspend in a desired volume of serum-free media and stored at $-80^{\circ} \mathrm{C}$.

\section{siRNA-mediated knockdown and pharmacological inhibition of KDM5D}

NCSCs were transduced with lentivirally packaged vectors produced as described above followed by verification of knockdown using qPCR (see below). Knockdown cells were seeded on collagen type I fibers and cultivated for 30 days as described above. Pharmacological inhibition of KDM5D was performed by exposing NCSCs to $1 \mu \mathrm{M}, 10 \mu \mathrm{M}$ and $100 \mu \mathrm{M}$ KDOAM-25 (KDOAM hydrochloride hydrate, SML2774, Sigma Aldrich) for 30 days of osteogenic differentiation on collagen type I fibers as described

\section{Total RNA was isolated as described above followed by cDNA synthesis using First Strand cDNA} Synthesis Kit (Fermentas, St. Leon-Rot, Germany). qPCR was performed using Platinum SYBR Green 
bioRxiv preprint doi: https://doi.org/10.1101/2021.11 10.468047; this version posted November 11, 2021. The copyright holder for this preprint (which was not certified by peer review) is the author/funder, who has granted bioRxiv a license to display the preprint in perpetuity. It is made available under aCC-BY-NC-ND 4.0 International license.

454 qPCR Super-Mix UDG (Invitrogen, Life Technologies GmbH, Darmstadt, Germany) according to the manufacturers guidelines and assayed with a Rotor Gene 6000 (QIAGEN, Hilden, Germany).

456 
457

458

459

460

461

462

463

464

465

466

467

468

469

470

471

472

473

474

475

476

477

478

479

480

481

\section{Acknowledgement}

We thank Elke Redeker and Angela Kralemann-Köhler for technical support. We thank the University of Bielefeld for supporting the study by a gender equality PhD stipend to MM and the Forschungsverbund BioMedizin Bielefeld FBMB for financing TN.

\section{Competing Financial Interests statement}

The authors declare no conflict of interest.

\section{Data availability statement}

The RNA-Seq datasets generated and analysed during the current study will be available in the NCBI's

Gene Expression Omnibus repository (48) through GEO Series accession number.

\section{Author contributions}

Madlen Merten: Investigation, data analysis and interpretation, writing and editing

Johannes F.W. Greiner: Investigation, data analysis and interpretation, writing and editing

Tarek Niemann: Investigation, data analysis and interpretation, writing and editing

Meike Grosse Venhaus: Investigation, data analysis and interpretation, writing and editing

Daniel Kronenberg: Data analysis and interpretation, editing

Richard Stange: Financing, editing

Dirk Wähnert: Investigation, data analysis and interpretation, editing

Christian Kaltschmidt: Data analysis and interpretation, writing and editing, financing

Thomas Vordemvenne: Financing, data analysis and interpretation, editing

Barbara Kaltschmidt: Conception and design, data analysis and interpretation, supervision writing and editing, financing 


\section{References}

1. Strube P, Mehta $M$, Baerenwaldt A, Trippens J, Wilson CJ, Ode A, et al. Sex-specific compromised bone healing in female rats might be associated with a decrease in mesenchymal stem cell quantity. Bone. 2009;45(6):1065-72.

2. Greiner J, Merten M, Kaltschmidt C, Kaltschmidt B. Sexual dimorphisms in adult human neural, mesoderm-derived, and neural crest-derived stem cells. FEBS Lett. 2019.

3. Riggs BL, Khosla S, Melton $L$, 3rd. Sex steroids and the construction and conservation of the adult skeleton. Endocrine reviews. 2002;23(3):279-302.

4. Møller AMJ, Delaissé J-M, Olesen JB, Madsen JS, Canto LM, Bechmann T, et al. Aging and menopause reprogram osteoclast precursors for aggressive bone resorption. Bone Research. 2020;8(1):27.

5. Pien DM, Olmedo DG, Guglielmotti MB. Influence of age and gender on peri-implant osteogenesis. Age and gender on peri-implant osteogenesis. Acta Odontol Latinoam. 2001;14(1-2):913.

6. Parker MJ, Raghavan R, Gurusamy K. Incidence of fracture-healing complications after femoral neck fractures. Clin Orthop Relat Res. 2007;458:175-9.

7. Cawthon PM. Gender differences in osteoporosis and fractures. Clin Orthop Relat Res. 2011;469(7):1900-5.

8. Ejiri S, Tanaka M, Watanabe N, Anwar RB, Yamashita E, Yamada K, et al. Estrogen deficiency and its effect on the jaw bones. J Bone Miner Metab. 2008;26(5):409-15.

9. Compston JE, McClung MR, Leslie WD. Osteoporosis. Lancet (London, England). 2019;393(10169):364-76.

10. Khosla S, Oursler MJ, Monroe DG. Estrogen and the skeleton. Trends in endocrinology and metabolism: TEM. 2012;23(11):576-81.

11. Zhou S, Zilberman Y, Wassermann K, Bain SD, Sadovsky Y, Gazit D. Estrogen modulates estrogen receptor alpha and beta expression, osteogenic activity, and apoptosis in mesenchymal stem cells (MSCs) of osteoporotic mice. J Cell Biochem Suppl. 2001;Suppl 36:144-55.

12. Meszaros LB, Usas A, Cooper GM, Huard J. Effect of host sex and sex hormones on musclederived stem cell-mediated bone formation and defect healing. Tissue Eng Part A. 2012;18(1718):1751-9.

13. Corsi KA, Pollett JB, Phillippi JA, Usas A, Li G, Huard J. Osteogenic potential of postnatal skeletal muscle-derived stem cells is influenced by donor sex. J Bone Miner Res. 2007;22(10):1592-602.

14. Aksu AE, Rubin JP, Dudas JR, Marra KG. Role of gender and anatomical region on induction of osteogenic differentiation of human adipose-derived stem cells. Ann Plast Surg. 2008;60(3):306-22.

15. Hauser S, Widera D, Qunneis F, Muller J, Zander C, Greiner J, et al. Isolation of novel multipotent neural crest-derived stem cells from adult human inferior turbinate. Stem Cells Dev. 2012;21(5):742-56.

16. Greiner JF, Gottschalk M, Fokin N, Buker B, Kaltschmidt BP, Dreyer A, et al. Natural and synthetic nanopores directing osteogenic differentiation of human stem cells. Nanomedicine. 2019;17:319-28.

17. Hofemeier AD, Hachmeister H, Pilger C, Schurmann M, Greiner JF, Nolte L, et al. Label-free nonlinear optical microscopy detects early markers for osteogenic differentiation of human stem cells. Sci Rep. 2016;6:26716.

18. Vordemvenne T, Wahnert D, Koettnitz J, Merten M, Fokin N, Becker A, et al. Bone Regeneration: A Novel Osteoinductive Function of Spongostan by the Interplay between Its Nano- and Microtopography. Cells. 2020;9(3).

19. Wähnert D, Koettnitz J, Merten M, Kronenberg D, Stange R, Greiner JFW, et al. Spongostan() Leads to Increased Regeneration of a Rat Calvarial Critical Size Defect Compared to NanoBone((R)) and Actifuse. Materials (Basel). 2021;14(8). 
20. Li K, Han J, Wang Z. Histone modifications centric-regulation in osteogenic differentiation. Cell Death Discovery. 2021;7(1):91.

21. Qin G, Li Y, Wang H, Yang J, Chen Q, Tang H, et al. Lysine-Specific Demethylase 4A Regulates Osteogenic Differentiation via Regulating the Binding Ability of H3K9me3 with the Promoters of Runx2, Osterix and Osteocalcin. Journal of biomedical nanotechnology. 2020;16(6):899-909.

22. Wang RN, Green J, Wang Z, Deng Y, Qiao M, Peabody M, et al. Bone Morphogenetic Protein (BMP) signaling in development and human diseases. Genes \& Diseases. 2014;1(1):87-105.

23. Choi J, Bae T, Byambasuren N, Park S-H, Jo CH, Kim D, et al. CRISPR-Cpf1 Activation of Endogenous BMP4 Gene for Osteogenic Differentiation of Umbilical-Cord-Derived Mesenchymal Stem Cells. Molecular Therapy - Methods \& Clinical Development. 2020;17:309-16.

542

543

544

545

546

547

548

549

550

551

552

553

554

555

556

557

558

559

560

561

562

563

564

565

566

567

568

569

570

571

572

573

574

575

576

24. Cheng SL, Shao JS, Charlton-Kachigian N, Loewy AP, Towler DA. MSX2 promotes osteogenesis and suppresses adipogenic differentiation of multipotent mesenchymal progenitors. J Biol Chem. 2003;278(46):45969-77.

25. Dacic S, Kalajzic I, Visnjic D, Lichtler AC, Rowe DW. Col1a1-driven transgenic markers of osteoblast lineage progression. J Bone Miner Res. 2001;16(7):1228-36.

26. Grigoriadis $A E$, Wang ZQ, Cecchini MG, Hofstetter W, Felix R, Fleisch HA, et al. c-Fos: a key regulator of osteoclast-macrophage lineage determination and bone remodeling. Science. 1994;266(5184):443-8.

27. Closs El, Murray AB, Schmidt J, Schön A, Erfle V, Strauss PG. c-fos expression precedes osteogenic differentiation of cartilage cells in vitro. J Cell Biol. 1990;111(3):1313-23.

28. Ohta S, Yamamuro T, Lee K, Okumura H, Kasai R, Hiraki Y, et al. Fracture healing induces expression of the proto-oncogene c-fos in vivo. Possible involvement of the Fos protein in osteoblastic differentiation. FEBS Lett. 1991;284(1):42-5.

29. Lee $\mathrm{W}-\mathrm{R}$, Ishikawa T, Umetani $\mathrm{M}$. The interaction between metabolism, cancer and cardiovascular disease, connected by 27-hydroxycholesterol. Clin Lipidol. 2014;9(6):617-24.

30. Schüle R, Siddique T, Deng HX, Yang Y, Donkervoort S, Hansson M, et al. Marked accumulation of 27-hydroxycholesterol in SPG5 patients with hereditary spastic paresis. Journal of lipid research. 2010;51(4):819-23.

31. Umetani M, Domoto H, Gormley AK, Yuhanna IS, Cummins CL, Javitt NB, et al. 27Hydroxycholesterol is an endogenous SERM that inhibits the cardiovascular effects of estrogen. Nat Med. 2007;13(10):1185-92.

32. DuSell CD, Nelson ER, Wang X, Abdo J, Mödder UI, Umetani M, et al. The Endogenous Selective Estrogen Receptor Modulator 27-Hydroxycholesterol Is a Negative Regulator of Bone Homeostasis. Endocrinology. 2010;151(8):3675-85.

33. Fagerberg L, Hallström BM, Oksvold P, Kampf C, Djureinovic D, Odeberg J, et al. Analysis of the human tissue-specific expression by genome-wide integration of transcriptomics and antibody-based proteomics. Molecular \& cellular proteomics : MCP. 2014;13(2):397-406.

34. Li L, Li Q, Wang Q, Liu L, Li R, Liu H, et al. Rare copy number variants in the genome of Chinese female children and adolescents with Turner syndrome. Bioscience Reports. 2019;39(1).

35. Faienza MF, Ventura A, Colucci S, Cavallo L, Grano M, Brunetti G. Bone Fragility in Turner Syndrome: Mechanisms and Prevention Strategies. Frontiers in Endocrinology. 2016;7(34).

36. Shang J, Wang H, Fan X, Shangguan L, Liu H. A genome wide analysis of alternative splicing events during the osteogenic differentiation of human cartilage endplate-derived stem cells. Mol Med Rep. 2016;14(2):1389-96.

37. Yin B, Yu F, Wang C, Li B, Liu M, Ye L. Epigenetic Control of Mesenchymal Stem Cell Fate Decision via Histone Methyltransferase Ash1l. STEM CELLS. 2019;37(1):115-27.

38. Yang $X$, Wang $G$, Wang $Y$, Zhou J, Yuan $H$, Li X, et al. Histone demethylase KDM7A reciprocally regulates adipogenic and osteogenic differentiation via regulation of $\mathrm{C} / \mathrm{EBP} \alpha$ and canonical Wnt signalling. J Cell Mol Med. 2019;23(3):2149-62.

39. Ge W, Shi L, Zhou Y, Liu Y, Ma G-e, Jiang Y, et al. Inhibition of Osteogenic Differentiation of Human Adipose-Derived Stromal Cells by Retinoblastoma Binding Protein 2 Repression of RUNX2Activated Transcription. STEM CELLS. 2011;29(7):1112-25. 
40. Hemming S, Cakouros D, Isenmann S, Cooper L, Menicanin D, Zannettino A, et al. EZH2 and KDM6A act as an epigenetic switch to regulate mesenchymal stem cell lineage specification. Stem Cells. 2014;32(3):802-15.

586 41. Feng Y, Wan P, Yin L. Long Noncoding RNA X-Inactive Specific Transcript (XIST) Promotes Osteogenic Differentiation of Periodontal Ligament Stem Cells by Sponging MicroRNA-214-3p. Medical science monitor : international medical journal of experimental and clinical research. 2020;26:e918932.

42. Ma X, Fan C, Wang Y, Du Y, Zhu Y, Liu H, et al. MiR-137 knockdown promotes the osteogenic differentiation of human adipose-derived stem cells via the LSD1/BMP2/SMAD4 signaling network. J Cell Physiol. 2020;235(2):909-19. represses ATF4-mediated transcription to regulate bone mass in transgenic mice. J Cell Biol. 2005;169(4):591-601.

596 44. Spicer PP, Kretlow JD, Young S, Jansen JA, Kasper FK, Mikos AG. Evaluation of bone regeneration using the rat critical size calvarial defect. Nat Protoc. 2012;7(10):1918-29. 3D cultivation method for adult human neural crest-derived stem cell therapeutics. Eur Cell Mater.

600 2011;22:403-19.

601 46. Gregory CA, Gunn WG, Peister A, Prockop DJ. An Alizarin red-based assay of mineralization by adherent cells in culture: comparison with cetylpyridinium chloride extraction. Anal Biochem. 2004;329(1):77-84.

604 47. Tang Y, Garson K, Li L, Vanderhyden BC. Optimization of lentiviral vector production using 605 polyethylenimine-mediated transfection. Oncology letters. 2015;9(1):55-62. hybridization array data repository. Nucleic acids research. 2002;30(1):207-10. 